\title{
Bilinguisme Et Developpement Cognitif A L'ecole Primaire. Une Etude Experimentale A Partir Du Terrain Ivoirien De La Region De Bondoukou
}

\author{
Seka Yapi Arsène \\ Th. Ecole Normale Supérieure d’Abidjan, département des Sciences de \\ l'Education. \\ Niamien Christiane \\ Université FHB d’Abidjan, département des Sciences du Langage.
}

doi: 10.19044/esj.2016.v12n22p134 URL:http://dx.doi.org/10.19044/esj.2016.v12n22p134

\begin{abstract}
Bilingualism is a subject of growing research in Ivory Coast since in many regions, there has been either a simultaneous bilingualism alternating bilingualism among school-age children. This sociological reality noted that children speak at least two local languages before school for learning French. Obviously, the young student who comes into the first primary level CM1), is facing a situation of multilingualism. From an experimental study, quantitative approach serves as a method of analysis to evaluate the effect of bilingualism among students of CM1 class of the Bondoukou region on academic performance. The study whose objective is indeed to make an analysis of the impact of bilingualism on cognitive development of learners, comes in operative assumptions. The results indicate a strong correlation between variables bilingualism, cognitive development and learning. The practice of two local languages in children proves to be an asset for cognitive development in general and learning in schools in particular. The performances by these schoolchildren in several disciplinary trials of their level, are justified in part by a good command of their native languages.
\end{abstract}

Keywords: Bilingualism, Cognitive Development, School learning, Mother tongue

\section{Résumé}

Le bilinguisme devient un objet d'étude de plus en plus important en Côte d'Ivoire vu que dans bon nombre de régions, on enregistre soit un bilinguisme simultané soit un bilinguisme alternatif chez les enfants scolarisables. Cette réalité sociologique fait remarquer que les enfants parlent au moins deux langues locales avant d'être scolarisés pour l'apprentissage du 
français. A l'évidence, le jeune apprenant qui entre en CP1, se trouve devant une situation de multilinguisme. A partir d'une étude expérimentale, l'approche quantitative sert de méthode d'analyse pour évaluer l'effet du bilinguisme chez des élèves des classes de CM1 de la région de Bondoukou sur le rendement scolaire. L'étude dont l'objectif vise en effet, à faire une analyse de l'impact du bilinguisme sur le développement cognitif des apprenants, se décline en hypothèses opératoires. Les résultats indiquent une forte corrélation entre les variables bilinguisme, développement cognitif et développement des apprentissages. La pratique de deux langues locales chez les enfants se révèle comme un atout pour le développement cognitif en général et celui des apprentissages dans le cadre scolaire en particulier. Les performances réalisées par ces écoliers dans plusieurs épreuves disciplinaires de leur niveau, se justifient en partie par une bonne maîtrise de leurs langues maternelles.

Mots clés : Bilinguisme, Développement cognitif, Apprentissages scolaires, Langue maternelle

\section{Introduction}

Le grand nombre de langues parlées en Côte d'Ivoire fait que le bilinguisme est un phénomène très fréquent aux niveaux social et individuel. Au sein de la population ivoirienne le bilinguisme se présente sous des formes différentes selon les caractéristiques du contexte sociolinguistique. Mais on peut dire, d'une manière générale, que le bilinguisme est associé au développement de certaines langues et au besoin d'acquérir des langues de communication intra-ivoirienne et nationale. Dans la plupart des régions du pays, le bilinguisme couvre des espaces géographiques importants et les enfants sont les premiers à montrer de l'intérêt à l'apprentissage d'une ou plusieurs langues tout autant qu'ils développent des performances réelles dans ces langues. Ces performances linguistiques sont révélatrices de la flexibilité des structures cognitives des enfants. Ainsi, l'intérêt de l'étude est suscité par la controverse selon laquelle le bilinguisme confère un avantage cognitif à ses locuteurs. En d'autres termes et dans l'esprit de l'étude, les locuteurs bilingues performent mieux que les monolingues dans plusieurs tâches. Quels sont les éléments qui permettent de soutenir ce point de vue. En admettant une telle hypothèse, l'étude qui se veut expérimentale, a donc pour objectif d'évaluer les performances des enfants bilingues et des enfants monolingues à partir de leurs productions scolaires. 


\section{Cadre theorique}

\section{Approche définitionnelle}

La littérature consacrée au bilinguisme a livré des définitions abondantes avec des nuances aussi bien dans la forme que dans le contenu du bilinguisme. Une approche définitionnelle semble donc nécessaire des notions de bilinguisme et de développement cognitif pour une compréhension du probable lien entre les variables. La notion de bilinguisme, selon Kloss et Mc Connel (1974) apparaît sous la plume du linguiste français Meillet Antoine en 1918. Elle désigne le fait de pratiquer deux langues autrement dit, une situation linguistique caractérisant des sujets parlant qui pratiquent concurremment deux langues. Pour Van (1972 :117118), "le bilinguisme consiste dans le fait de pouvoir s'exprimer sans difficulté dans deux langues à un degré de précision identique dans chacune d'elle; c'est la mise en présence de deux langues qui provoque un ensemble d'interférences linguistiques, psychologiques et sociologiques, susceptibles de déterminer un conflit de langage et donc d'identité. Il s'agit de l'usage alternatif de deux idiomes que le sujet parlant emploie tour à tour pour les besoins de son expression."

Le bilinguisme est l'emploi actif et passif de deux langues de statut identique par un locuteur.

Il consiste théoriquement dans le fait de pouvoir s'exprimer et penser sans difficulté dans deux langues. Les individus authentiquement bilingues sont imprégnés des deux cultures indifféremment dans tous les domaines. En d'autres termes, il s'agit de la compétence de locuteur natif dans deux langues. Pour le célèbre linguiste Bloomfield (1933), le bilinguisme est la possession d'une compétence de locuteur natif dans deux langues. Cette conception du bilinguisme plus ou moins populaire relève d'une certaine exception chez Mac Namara (1966) pour qui ce concept renferme chez le locuteur bilingue, la capacité de comprendre, parler, lire et écrire les deux langues concernées. Si la présence de deux langues semble faire l'unanimité chez le bilingue, les divergences de vue s'alimentent surtout du point de vue du contenu du concept. Ainsi, de l'analyse de Titone (1972) sur le bilinguisme, il ressort que: le bilinguisme consiste en la capacité d'un individu de s'exprimer dans une seconde langue en respectant les concepts et les structures propres à cette langue, plutôt qu'en paraphrasant sa langue maternelle. Le sujet bilingue s'exprime donc dans n'importe laquelle des deux langues sans véritable difficulté lorsque l’occasion se présente.

Les recherches sur le concept de bilinguisme se sont multipliées au fil des années ouvrant ainsi des voies nouvelles sur l'étendue de la signification du phénomène. Ainsi, avec Mackey, (1976), la conception du bilinguisme retrouve une originalité différente des autres. Le bilinguisme se décline pour lui comme l'usage alterné de deux ou plusieurs langues par le même 
individu. Selon l'auteur, le bilinguisme se détermine par quatre niveaux que sont le degré : la connaissance que l’individu possède des deux langues qu'il emploie, la fonction : le rôle que ces langues jouent dans la structure globale de son comportement et les buts visés par l'usage de ces langues, l'alternance : les conditions et la manière permettant le passage d'une langue à l'autre et l'interférence : la condition dans laquelle l'individu bilingue arrive à maintenir les deux langues séparées. Beardsmore (1987), quant à lui, établit une différence entre bilinguisme social qui fait appel à la compétence de tout le corps social et bilinguisme individuel.

Les travaux de Dulce Rebelo (2000), se sont orientés sur la typologie du bilinguisme. Trois types du bilinguisme sont à prendre en compte : le bilinguisme coordonné quand les signes de chaque langue combinant une unité de sens et une unité d'expression sont maintenus séparés; le bilinguisme composé quand les signes combinent une seule unité de sens avec une unité d'expression dans chaque langue. Le bilinguisme subordonné, quand le signifié de la langue maternelle correspond à deux signifiants : l'un dans la langue maternelle, ce qui est bien, l'autre, inapproprié dans la deuxième langue. Le bilinguisme constitue la forme la plus simple du multilinguisme et s’oppose au monolinguisme. Une personne bilingue, dans le sens le plus large, défend Caliari, (2011) est celle qui peut communiquer dans deux langues, que ce soit sous forme active (parole, écriture) ou passive (écoute, lecture). Certains linguistes plaident pour une définition maximale signifiant que les vrais bilingues sont aussi bien capables de s'exprimer dans une langue que dans l'autre et qu'ils ont une connaissance identique des deux langues. D’autres plaident pour une définition minimale’', basée sur l'utilisation correcte de phrases dans les deux langues pour la communication courante.

\section{Problématique}

Ces différentes typologies du bilinguisme telles que présentées par leurs auteurs, intègrent de façon significative la situation linguistique de la zone d'expérimentation. Mais l'accès à un cadre de référence clair et mieux adapté aux besoins de l'étude d'une part et le concept de bilinguisme luimême qui échappe à une définition précise d'autre part, nous conduit à nous pencher plus, sur les effets du bilinguisme sur le développement cognitif de l'échantillon d'étude que sont les apprenants des classes du primaire des cours moyens 1 (CM1), représentant la cinquième année du primaire.

La grande majorité des élèves dans la région de Bondoukou, sont bilingues. Il s'agit d'un bilinguisme à la fois social et individuel qui se caractérise par le contact de deux communautés linguistiques différentes aussi bien par leurs cultures que par la nature de leurs langues respectives. 
L'une appartient à l'ère Gur ${ }^{32}$ tandis que l'autre fait partie du grand groupe $\mathrm{Kwa}^{33}$. Les langues Koulango et Abron logées dans le Nord-Est de la Côte d’Ivoire, sont deux langues qui ont favorisé un bilinguisme au seul profit des populations Abron. En effet, ils sont les seuls dans toute la région à parler aussi bien leur langue maternelle que le Koulango. Le contact des deux communautés entretenu par des interactions de toutes sortes et marqué par une parfaite maîtrise de la langue voisine par le peuple Abron, suscite beaucoup d'intérêt pour la recherche en psycholinguistique.

En effet, comment des enfants bilingues parviennent-ils à réaliser des performances scolaires jusqu'à devenir des multilingues puisqu'ils devront connaître le français et plus tard, l'anglais et d'autres langues encore. De quelle manière, le bilinguisme favorise-t-il une telle habileté du cerveau alors que des études menées antérieurement par Pintner et Keller (1922) soulignent le caractère nocif du bilinguisme sur le développement intellectuel de l’apprenant. Ils sont soutenus dans leur thèse par Saer (1923) qui atteste que chez l'enfant bilingue, le fonctionnement intellectuel se caractérise par une confusion mentale lors des apprentissages scolaires. Cependant, l'état actuel des recherches nous informent sur les avantages cognitifs du bilinguisme chez l'enfant. A partir d'une expérimentation du terrain ivoirien dans la région de Bondoukou réputée pour son bilinguisme, nous nous emploierons à rechercher l'impact des variables bilinguisme et monolinguisme sur les rendements scolaires des apprenants.

Si pour des raisons culturelles et sans doute historiques que nous ignorons, le peuple Abron a délibérément adopté la langue Koulango sans toutefois négliger sa langue maternelle, l'intérêt de l'étude réside moins à comprendre l'inféodation linguistique d'un peuple à un autre mais plutôt les avantages psychologiques en termes de cognition dont jouirait les bilingues. La région de Bondoukou, zone d'expérimentation de l'étude, regorge de nombreux enfants dont les performances cognitives séduisent l'imagination populaire. De l'habileté linguistique caractérisée par le passage d'une langue à une autre, au raisonnement qu'ils développent face à une situation problème, en considérant tout aussi bien leurs résultats scolaires, force est de s’interroger sur un probable lien entre toutes ces variables.

Aussi, la question fondamentale qui oriente ce travail est celle de rechercher des explications qui relient les variables en présence. En d'autres termes, la capacité chez l'enfant à parler plusieurs langues influence-t-elle sa cognition ou alors favorise-t-elle son développement cognitif. Il est entendu que le cognitif ici, est l'ensemble de ce qui relève de la cognition c'est-à-

\footnotetext{
${ }^{32}$ Gur: Terme pour désigner l'ensemble des groupes ethniques issus du Nord de la Côte d'Ivoire.

${ }^{33}$ Kwa: Terme pour désigner l'ensemble des groupes ethniques issus du Sud et de l'Est de la Côte d'Ivoire.
} 
dire, l'ensemble des processus mentaux qui se rapportent à la fonction de connaissance telle que la mémoire, le langage, le raisonnement, l'apprentissage la résolution de problème. Ces processus mentaux qui se réaliseraient plus aisément chez les enfants bilingues, conduisent à mener une réflexion sur le bilinguisme et le développement cognitif chez les écoliers. Le bilinguisme a-t-il un effet sur le développement cognitif des enfants ? Ou encore, le bilinguisme est-il un accélérateur de l'intelligence de l'enfant ? En supposant que cette hypothèse est vraie, quelles sont dans le cadre des apprentissages scolaires, les différentes disciplines qui sont directement influencées par la pratique et la maîtrise de deux langues ? Il convient dans l'étude, de considérer que les langues que parlent les enfants et qui sont objet de bilinguisme, sont des langues locales, développées en dehors du cadre scolaire.

\section{Methodologie}

La méthodologie repose sur une démarche expérimentale et opérationnelle. Par son caractère expérimental, l'étude s'inscrit dans les méthodes qualitative et quantitative. Formuler en effet, l'hypothèse selon laquelle l'enfant bilingue performe mieux que le monolingue en termes de rendement scolaire, c'est chercher à établir une relation de cause à effet entre le bilinguisme et le développement cognitif chez les enfants apprenants. Comparer la performance des élèves bilingues à celle des monolingues, c'est déterminer un groupe expérimental (bilingues) et un groupe de contrôle (monolingues). Le caractère opérationnel réside en ce que les résultats du travail permettront de féconder les réflexions et de dynamiser les politiques d'enseignement en Côte d'Ivoire.

\section{Objectifs et Hypothèses \\ Objectif général}

L'étude vise à analyser l'effet du bilinguisme sur le développement cognitif des enfants scolarisés de la région de Bondoukou.

\section{Objectifs spécifiques}

- $\quad$ Décrire les conséquences du bilinguisme dans le développement de l'enfant.

- $\quad$ Evaluer l'effet du bilinguisme sur les disciplines scolaires.

1-2/ Hypothèse générale : Le bilinguisme impact sur le développement cognitif des apprenants et sur leurs rendements scolaires par extension. 


\section{Hypothèses spécifiques :}

- $\quad$ Il existe un lien entre le bilinguisme et les apprentissages chez l'enfant. scolaires.

Le bilinguisme de l'enfant favorise de meilleures performances

Echantillon, Instruments, Méthode d'analyses et traitement des données.

\section{Taille et composition de l'échantillon}

L'échantillon actif est composé de 150 sujets répartis comme suit : 75 sujets à Bondoukou à l'EPP du château et 75 autres aux EPP Tabagne et Kwassi Ndawa. L'enquête se fait à partir de deux localités distinctes. La ville de Bondoukou est un département situé dans le sud Est de la Côte d'Ivoire où toutes les fonctions de l'administration centrale sont représentées. Tabagne et Kwassi Ndawa sont deux villages situés respectivement à 20 et $22 \mathrm{Km}$ de la ville. Les seuls agents de l'Etat sont les instituteurs. IL n'y a pas d'école maternelle. La population est paysanne et l'agriculture manuelle est l'activité principale.

\section{Instrument de recueil des données}

Le principe est d'évaluer chez l'enfant, son niveau de connaissance dans les disciplines suivantes :

\section{Grammaire}

Les filles se sont (mettre) à jouer

Les mangues sont (tomber) dans la cour

Qu'elles sont belles les maisons que nous avons (construire).

\section{Mathématique}

Koffi, Ama et Micheline produisent ensemble une tonne (1000kg) de caoutchouc (latex). Koffi a produit $150 \mathrm{~kg}$ de plus qu'Ama. Ama a produit deux fois la quantité produite par micheline.

Fais une représentation graphique des quantités produites par chacun.

\section{Vocabulaire}

Trouvez un synonyme des mots ou expressions suivantes.

Un agriculteur, obéir à ses parents, content, riche, se sentir mal.

Rédaction raconte en quelques lignes une fête à laquelle tu as participé.

Les notes assorties de ces devoirs constituent l'unité de mesure. L'enfant sera jugé excellent dans ces disciplines s'il a une moyenne supérieure ou égale à dix. 


\section{Outil et Méthode d'analyse des données}

L’observation est centrée sur les élèves bilingues et monolingues en situation de classe. Il s'agit de les regarder composer. Nous pouvons également observer à travers la même séance, le comportement de l'élève pendant que le maître fait une leçon. L’entretien n’est pas exclu ; il permet de constater la réaction d'un apprenant et son analyse d'une situation pendant une conversation. Les méthodes quantitatives et qualitatives sont sollicitées dans cette étude.

\section{Traitement des données}

Le traitement se fera sur à partir des notes recueillies après évaluation des apprenants. Les résultats du traitement traduisent l'impact des variables (bilinguisme/monolinguisme) sur les rendements des enfants. Le SPSS (statistical package for social sciences) est le logiciel de traitement de données en vue d'analyses statistiques. Nous procédons à l'inscription des variables indépendantes dans les cellules de tableaux; ensuite, nous enregistrons successivement les variables dépendantes que sont les différentes performances aux différentes épreuves subies. A ces performances, nous procédons à un système de codage : le chiffre 1 est affecté aux enfants qui réussissent aux épreuves et le chiffre 0 affecté à ceux des enfants qui ne réussissent pas l'épreuve. Ce système a été valable tant pour toutes les disciplines. Enfin, après avoir enregistré toutes ces données, nous lançons l’opération et obtenons les résultats statistiques, (les khi-deux, les mesures, et tableaux, que nous commentons dans une série d’analyses.

\section{Resultats}

\begin{tabular}{|c|c|c|}
\multicolumn{1}{c|}{ Tableau 1: bilinguisme / production lexicale en français } \\
\cline { 2 - 3 } & Moyenne $\geq 10$ en production lexicale française \\
\cline { 2 - 3 } & oui & non \\
\hline bilingues (N=75) & 70 & 5 \\
& $93,34 \%$ & $6,66 \%$ \\
\cline { 2 - 3 } monolingues (N=75) & 18 & 57 \\
\hline Total & $24 \%$ & $76 \%$ \\
\hline 150 & 88 & 62 \\
\hline
\end{tabular}

Les bilingues ont un niveau lexical plus élevé que les monolingues. On enregistre en effet $93,34 \%$ de taux de succès pour les bilingues contre $24 \%$. Le bilinguisme semble avoir un effet sur la production lexicale des enfants. Le test d'indépendance des variables bilinguisme et performance lexicale chez les enfants est significatif au seuil de $5 \%$ car la valeur du khi deux calculée $(71,508)$ est supérieure à la valeur tabulée à 1 ddl $(3,841)$. Le 
lien entre bilinguisme et performance lexicale est donc confirmé ce qui veut dire que l'hypothèse selon laquelle le bilinguisme a un effet sur un meilleur niveau lexical en français chez les enfants est vérifiée.

La valeur du coefficient de contingence $\Phi=0,704$ confirme la très forte liaison entre les deux variables.

Tableau 2 : bilinguisme / épreuve de mathématique.

\begin{tabular}{|c|c|c|}
\cline { 2 - 3 } \multicolumn{1}{c|}{} & \multicolumn{2}{c|}{ Moyenne $\geq 10$ à l'épreuve de mathématique } \\
\cline { 2 - 3 } \multicolumn{1}{c|}{} & oui & non \\
\hline bilingues & 64 & 11 \\
$\mathrm{~N}=75$ & $85,33 \%$ & $14,67 \%$ \\
\hline monolingues & 53 & 22 \\
$\mathrm{~N}=75$ & $70,67 \%$ & $29,33 \%$ \\
\hline Total & 117 & 33 \\
150 & $78 \%$ & $22 \%$ \\
\hline
\end{tabular}

L'examen du tableau montre que les performances des bilingues (85,33\%) sont plus fortes que celles des monolingues (70,67\%). Le bilinguisme semble avoir un lien sur les performances des enfants. Le test d'indépendance du $\mathrm{X}^{2}$ des variables bilinguisme et prononciation des syllabes inversées est significatif car la valeur du $X^{2}$ calculée au seuil de 5\% à $1 \mathrm{ddl}(4,701)$ est supérieure à la valeur du khi deux tabulée $(3,841)$ à $1 \mathrm{ddl}$. Nous pouvons conclure pour dire que le lien entre le bilinguisme et la prononciation des syllabes inversées est significatif d'où, l'hypothèse selon laquelle le bilinguisme favorise des compétences en prononciation de syllabes inversées est vraie. Cependant, ce lien reste encore très faible, au regard de la valeur du coefficient de contingence $\Phi=-0,177$.

Tableau 3 : bilinguisme / épreuve de grammaire.

\begin{tabular}{|l|l|l|}
\cline { 2 - 3 } \multicolumn{1}{c|}{} & \multicolumn{2}{c|}{ Moyenne $\geq 10$ à l'épreuve de grammaire } \\
\cline { 2 - 3 } \multicolumn{1}{c|}{} & oui & non \\
\hline bilingues & 51 & 24 \\
& $68 \%$ & $32 \%$ \\
\hline monolingues & 43 & 32 \\
N=75 & 43 & $42,67 \%$ \\
\hline $\begin{array}{l}\text { Total } \\
150\end{array}$ & $57,33 \%$ & 56 \\
\hline & 94 & $37,33 \%$ \\
\hline
\end{tabular}

On note un taux de réussite de $68 \%$ chez les bilingues. Chez les monolingues, nous enregistrons 57,33\% de réussite.

La différence constatée entre les groupes d'enfants n'est pas significative au seuil de $5 \%$ à 1 degré de liberté car le khi carré expérimental $(0,11)$ est inférieur au khi carré tabulé $(3,841)$. On ne peut donc pas affirmer que la performance à l'épreuve de grammaire est liée au bilinguisme de 
l'enfant. Il est à noter tout de même que les bilingues sont plus nombreux à développer des performances en grammaire.

Tableau 4 : Bilinguisme /expression écrite.

\begin{tabular}{|l|l|l|}
\cline { 2 - 3 } \multicolumn{1}{c|}{} & \multicolumn{2}{l|}{$\begin{array}{l}\text { Moyenne } \geq 10 \text { à l'épreuve de l'expression } \\
\text { écrite en français }\end{array}$} \\
\cline { 2 - 3 } \multicolumn{1}{c|}{} & oui & non \\
\hline $\begin{array}{l}\text { Bilingues } \\
=75\end{array}$ & $\begin{array}{l}41 \\
54,6 \%\end{array}$ & $\begin{array}{l}34 \\
45,3 \%\end{array}$ \\
\hline $\begin{array}{l}\text { Monolingues } \\
75\end{array}$ & 13 & 62 \\
\hline total=150 & $17,3 \%$ & $82,7 \%$ \\
& 54 & 96 \\
\hline
\end{tabular}

Les bilingues affichent de meilleures performances. On enregistre en effet $(63,3 \%)$ de réussite tandis que chez les monolingues $(17,3 \%)$ sont annoncés.

Le test d'indépendance des variables des bilingues et les performances aux épreuves d'expression écrite est significatif au seuil de 5\% et même au seuil de $1 \%$; car la valeur calculée du $X^{2}(30,006)$ à 1 degré de liberté est supérieure à la valeur tabulée $(3,841)$.

De plus, le Coefficient de contingence $\Phi=0,426$ indique que le lien est assez fort entre le bilinguisme des élèves et leur performance à l'expression écrite.

\section{Discussion}

\section{Le bilinguisme: un stimulant à l'apprentissage}

L'hypothèse selon laquelle le bilinguisme peut avoir un impact sur le développement cognitif chez les enfants est controversée par le courant constructiviste de Piaget (1962). Il reconnaît cependant que les structures cognitives qui se développent chez ces derniers, sont la conséquence d'une série d'organisations qui se matérialisent par l'activité personnelle des enfants. Mais, en reconnaissant l'évolution des structures cognitives vers une pensée de plus en plus logique, on se demande comment la pensée peut se structurer sans le langage.

L'étude expérimentale faite à partir de deux groupes d'enfants (bilingues et monolingues) révèle un lien du bilinguisme au développement de leurs performances scolaires. En d'autres termes, il convient de souligner que plus les enfants parlent deux langues, davantage ils présentent de meilleurs résultats. Le développement des compétences scolaires chez l'enfant est dans une large mesure, influencé par des facteurs 
d'environnement; la diversité communicationnelle sous le rapport des interactions humaines et sociales. Le contexte familial, l'intelligence exercent une forte influence sur le développement de la capacité d’apprendre chez l'enfant.

Les étapes successives du développement cognitif bénéficient du rôle primordial de l'expérience comme moteur de la construction ou de la reconstruction au niveau des représentations internes de l'enfant. Cette expérience, dans notre cas ici, est matérialisée par l'usage chez les enfants de deux langues avec des structures de fonctionnement différentes. La pratique de deux langues en situation d'apprentissage stimule davantage le cerveau humain ce qui fait dire Andrillon (1964:60) "chez les intellectuels, le cerveau a de gros besoins d'oxygène. Les efforts cérébraux provoquent un afflux de sang au cerveau, les cellules cérébrales sous tension, font appel à une circulation sanguine intense". Notre intérêt aux propos de cet auteur est moins l'oxygénation du cerveau que l'intensité de l'activité que celui-ci mène chez certaines personnes. En effet, l'usage de deux langues différentes est un travail hautement intellectuel qui met en présence deux modes de pensée différents dans un processus d'accommodation qui conduit inéluctablement la pensée primitive à une équilibration majorante; c'est-àdire, à un développement de la connaissance intellectuelle donc du développement cognitif. C’est d'ailleurs à juste titre que Tresse (2014:1) affirme ceci : "...plus l'activité cérébrale est intense, plus les capacités du cerveau continuent à se développer ».

L'équilibration des structures cognitives traduit le passage d'un état de moindre équilibre résultant de la prestation du sujet aux perturbations extérieures vers un équilibre supérieur correspondant à des possibilités nouvelles grâce à une structure cognitive plus forte. Le modèle Piagétien du développement cognitif, offre un cadre de réflexion utile à l'éducation par la mise en évidence du rôle bénéfique du conflit cognitif même si l'auteur, exclut les systèmes de représentations que sont le langage entre autres.

Le langage traduit la pensée humaine; il est comme l'indique Segalowitz (1977 :135), " une sorte de calcul mental, capable de manipuler les symboles de la pensée ». Il ne peut être postérieur ni antérieur à celle-ci. Langage et pensée sont deux facultés intimement liées qui constituent les deux faces d'une même pièce. A cet effet, Dulce Rebelo (2000: 2 ), soutiendra que : "l'intervention du langage est nécessaire car il apporte une contribution utile à l'expression de la pensée abstraite » De son côté, Vygotski (1962) soutient la thèse selon laquelle le langage joue un rôle important dans le développement cognitif. Instrument psychologique de premier ordre, le langage occupe une place exemplaire dans le schéma développemental de l'intelligence. Le langage devient pour l'enfant, un moyen pour organiser ses propres actions sur le monde. La portée de la 
théorie Vygotskienne confère au langage une dimension transversale à toute opération cognitive; c’est un instrument de régulation des activités mentales.

Le langage intériorisé influence le développement cognitif car il est à l'origine d'un système abstrait qui permet l'organisation de la pensée. Dans le cas du bilinguisme, dira Rebelo (2000:1), «...il y aura l'intériorisation de deux langues c'est-à-dire une représentation plus élaborée qui permet l'alternance entre deux systèmes de règles dans la manipulation des symboles ». Le développement cognitif chez les bilingues s'explique donc doublement dans la mesure où il existe chez eux, deux systèmes de langues différents qui rendent le cerveau plus actif et plus flexible dans un processus d'apprentissage. C'est aussi ce que pense Abdelilah-Bauer (2015 :38) quand elle soutient que: "les bilingues(...) ont une pensée plus créative, plus flexible et plus ouverte. C'est ce qu'on appelle la pensée divergente qui produit des solutions originales et moins conventionnelles. Les études qui ont établi cela partent de l'hypothèse que le fait d'avoir deux systèmes de représentation mentale augmente la flexibilité et l'originalité de la pensée...».

Le développement cognitif, il est vrai, répond à un processus d'auto construction qui vise à organiser la pensée chez l'enfant. Mais il est évident que l'enfant n’organise sa pensée qu’à partir de la langue qu'il connait. Wallon (1970), un défendeur de l'importance des systèmes de représentations comme le langage dans le développement intellectuel, soutient que le développement de l'enfant doit intégrer les aspects intellectuels et les aspects sociaux communicatifs. Le langage est la conséquence du développement social et du développement intellectuel; il n'est pas la cause de la pensée mais l'instrument, le support indispensable à ses progrès. Selon cette position, l’évolution du langage est liée aux capacités motrices et sensorimotrices de l'enfant. Le langage apparait vers 2 ans et dès ce moment, il commande et structure la pensée qui, initialement liée à l’action, se socialise grâce et par le langage.

\section{Bilinguisme et rendement scolaire}

Si l'action du langage est aussi central dans le développement cognitif de l'enfant, il est sans surprise que des enfants exposés à deux langues qu'ils pratiquent au quotidien auront un avantage cognitif sur des monolingues. En effet, dans le milieu scolaire, les bilingues réalisent en général de meilleures performances moyennes que les monolingues relativement aux compétences préalables aux apprentissages fondamentaux. Une telle différence traduit l'existence d'un lien significatif entre le bilinguisme et le développement cognitif qui s'observe à travers les performances scolaires des enfants. Le bilinguisme a un effet positif sur le développement des capacités des enfants en production lexicale, en 
mathématique en grammaire et en expression écrite. Les performances réalisées par les bilingues attestent de l'avantage cognitif de ceux-ci par la pratique de plusieurs langues.

Tout commence par une attention plus sélective et une meilleure concentration chez le bilingue. Les enfants bilingues précoces sont capables de fixer leur attention sur un objectif précis et inhiber les éléments perturbateurs. Ainsi, les performances réalisées en grammaire et en mathématiques sont révélatrices du développement cognitif entretenu par le bilinguisme. Dans une étude menée à l'université Concordia à Montréal, Poulin-Dubois (2011) soutient en effet que les aires cérébrales dévolues au langage (l'aire de Broca associée à la production du langage et l'aire de Wernicke associée au traitement des paroles entendues) et leur développement sont identiques chez les bilingues et les monolingues. La différence tient au fait que le lobe frontal (notamment associé dans l'élaboration de processus cognitifs complexes tels que la planification ou le raisonnement déductif) est plus sollicité chez les bilingues.

Il n’y a aucun doute, que la grammaire et les mathématiques sont deux disciplines scolaires qui procèdent d'un raisonnement logique, d'une démarche méthodique et bien hiérarchisée. De ce fait, le lobe frontal dont la sensibilité aux raisonnements logiques reste la principale fonction, libère les substances appropriées pour la mise en place d'un processus de compréhension du problème à résoudre. Le cerveau du bilingue, contrairement au monolingue, est plus habitué à la planification et au raisonnement par le développement de deux systèmes linguistiques différents. Cette habileté lui confère une certaine plasticité qui n’est pas étranger au lobe frontal. Les informations parviennent au lobe par le biais des neurones et les circuits nerveux se modifient plus facilement chez les bilingues. Tresse $(2014: 1)$ dira à ce propos : "plus on fait travailler certaines connexions plus elles sont performantes, moins on les fait travailler plus elles se détériorent. Par exemple, si vous apprenez un nouveau mot, de nouvelles connexions se créent. Plus vous utilisez ce mot, plus le chemin d'accès à ce mot dans votre cerveau s'améliore et plus vous le retrouvez facilement ». Les bilingues réussissent plus aux épreuves de grammaire et de mathématique pour la bonne raison que le lobe frontal s'est développé avec la pratique constante d'exercices de planification dus à la présence permanente de deux langues dans le cerveau. Le bilinguisme développe chez l'enfant, une conscience morphosyntaxique, lui permettant de comprendre plus tôt, les règles de grammaire.

Les résultats assortis de l'étude suggèrent que les enfants bilingues présentent une capacité cognitive supérieure. Ils développent une meilleure attention qui, en situation d'apprentissage scolaire, leur permet de mieux se concentrer en faisant abstraction de tout stimulus perturbateur. Morales 
(2013), aborde dans la même idée pour dire que : "le bilinguisme permet non seulement d'améliorer la mémoire de travail de façon isolée, mais améliore aussi le développement des fonctions exécutives. L'attention sélective et la flexibilité cognitive sont deux aspects importants de la cognition et plus particulièrement des habiletés liées aux fonctions exécutives ». La communication orale ou écrite étant une fonction exécutive, il est plus aisé de comprendre et d'expliquer les performances des bilingues en vocabulaire et en expression écrite. Le bilinguisme favorise chez les enfants, un meilleur apprentissage de la langue française à l'école. Au début de sa scolarisation, le jeune bilingue ne connaît pas le français. Le désir de parler le français d'une part et la flexibilité du cerveau d'autre part, due à la présence de deux langues, vont exiger de lui une grande attention.

Attention et flexibilité vont interagir pour accélérer l'apprentissage du français. Les habiletés deviennent plus fortes chez l'apprenant et les performances s'actualisent. Le bilinguisme favorise ce qui convient d'appeler une auto régulation, qui ouvre ainsi la porte à certaines habiletés chez l'enfant. Il donne, comme le dit Colombant (2011) «... Les enfants bilingues ont des avantages intellectuels, plus de souplesse mentale, plus de créativité, plus d'originalité dans leur façon de penser, trouvent plus facilement deux ou trois solutions à un problème. Ils peuvent trouver plus facilement beaucoup de réponses à une question ou un problème». Les performances enregistrées chez les apprenants bilingues en vocabulaire aussi bien qu'en expression écrite, témoignent de «la capacité pour le bilingue de passer d'une langue à une autre surtout les langues qui nécessitent de décaler son intérêt d'un item à l'autre ». Bialystok (2007). Pearson (1997) pense la même chose. Pour lui, le bilinguisme a un effet sur les apprentissages scolaires et il soutient qu'en combinant le vocabulaire total des langues parlées par les bilingues, ils ont habituellement un vocabulaire plus grand que les monolingues.

\section{Conclusion}

L’objectif de départ est de faire une analyse de l'effet du bilinguisme sur le processus cognitif des enfants apprenants des classes de cm1. L'exploitation des résultats donne lieu de comprendre que l'hypothèse selon laquelle le bilinguisme a un effet considérable sur les apprentissages scolaires et sur le développement cognitif en général est vraie. Contrairement à ces nouvelles révélations, d'anciennes théories incarnées par des auteurs comme Pintner (1922) soutenaient que les enfants bilingues souffraient d'un handicap linguistique et présentaient de ce fait, le bilinguisme comme un obstacle au développement intellectuel de l'enfant. Les études et recherches actuelles démontrent le contraire et attestent que le bilinguisme, selon JaphaAdi (2010), améliore la créativité chez les enfants ; quand Westly (2011) 
découvre à travers des études que le bilinguisme permet de développer des facilités d'adaptation précoces. Outre les recherches déjà effectuées sur la question, l'étude expérimentale que nous avons menée à partir d’un bilinguisme local c’est-à-dire, un bilinguisme qui s’est développé à partir de deux langues nationales ivoiriennes, montre que l'existence d'un lien entre bilinguisme et développement cognitif est irréfutable. Les politiques éducatives devraient alors encourager à la pratique de plusieurs langues dans nos écoles en considérant, la langue maternelle comme substrat linguistique dans l'enseignement.

\section{References:}

ABDELILAH, B. (2015). Le défi des enfants bilingues. Grandir et vivre en parlant plusieurs langues. La découverte. France.

ANDRILLON, M. (1964). Comment défendre et cultiver son cerveau. Ed, la Diffusion Nouvelle du Livre. Paris.

BEARDSMORE, B, H., (1987). Contact de langues : quels modèles. Dans Langue et Société, $\mathrm{n} 1,21$.

BIALYSTOK, E. (2007). Cognitive effects of bilingualism. How linguistic experience leads to cognitive change. International of Bilingual Education and Bilingualism, 10, 210-223. Canada.

BLOOMFIELD, L. (1933). Language. New York. Holt

CALIARI, P. (2011). Bilinguisme et apprentissage précoce des langues. Repéré dans www.peddycaliari.com Ouvert le 13 Juillet 2016.

COLOMBANT, K., (2011). Les avantages du bilinguisme. Repéré dans www.francais-du-mond.org, le 16 juillet 2016.

JAPHA-ADI, E. (2010). Cognitive Flexibility in drawings of bilingual children. In child development. 81(5) 1356-66 Bar Ilan University.

KLOSS, H., \& MC CONNEL, G., D., (1974). Composition linguistique des nations du monde. L'Asie du sud, secteur central et occidental. Vol 1. Press Universitaire Laval. Canada.

MAC NAMARA, J. (1966). Bilinguism and Primary Education. Edinburgh University Press.

MACKEY, W. (1976). Bilinguisme et contact des langues. Paris. Editions Klincksieck.

PEARSON, B.Z., (1997). The relation of input factors to lexical learning by bilingual infant. Applied psycholinguistics, 18, 41-58.

PIAGET, J. (1962). Le langage et les opérations intellectuelles. Dans problèmes de psycholinguistique. Paris presses universitaires. 51-61

PINTNER, R. \& KELLER, R., (1922). Intelligence tests for foreign children. Journal of educational psychology 
POULIN- DUBOIS, D., BLAYE, A., (2011). The effects of bilinguism on toddlers'executive functioning. Journal of Experimental Child Psychology, 108, 567-579. Ontario

REBELO, R. (2000). Bilinguisme et fonctionnement cognitif. Revue de l’Université de Lisbonne. 581, 579-580.

SAER, O., J., (1923). The effect of bilingualism on intelligence. British Journal of psychology. 14, 25-28.

SEGALOWITZ, N. (1977). Psychological perspectives on bilingual education. In Spolsky, R cooper eds. Frontiers of bilingual education. Rowley, Massachusetts Newbury house.

TITONE, R., (1972). Le bilinguisme précoce. Bruxelles. Dressart.

TRESSE, J. (2014). Les avantages du bilinguisme. Repéré dans www.educavox.fr Ouvert le 12 juillet 2016.

VAN, O, M., (1972). Introduction au problème du bilinguisme. Langue et culture, Paris, Labor.

VYGOTSKI, S. (1962). Thought and language. Cambridge, Massachusetts, Usa: MIT Press.

WALLON, H., (1970). De l'acte à la pensée. Paris : Flammarion.

WESTLY, E. (2011). Enfants bilingues : un avantage indéniable. Repéré dans www.cerveauetpsycho.fr Ouvert le 16 juillet 2016. 